\title{
An experimental study on restoration technique for the Acropolis of Athens: developing the experimental set-up for pull-out testing of titanium bars embedded in marble blocks
}

\author{
Ioanna Dakanali \\ Laboratory of Testing and Materials, Department of Mechanics, School of Applied Mathematical and Physical Sciences, \\ National Technical University of Athens, Zografou Campus, 15773 Athens, Greece \\ dakanalioanna@gmail.com
}

\author{
Aikaterini Marinelli \\ School of Engineering and the Built Environment, Edinburgh Napier University, \\ Merchiston Campus, EH10 5DT Edinburgh, Scotland \\ A.Marinelli@napier.ac.uk
}

\begin{abstract}
This work aims at investigating the mechanisms activated during the extraction of titanium bars from a marble volume (pull-out phenomenon), used for rejoining fragmented marble structural members at the monuments of the Acropolis of Athens. The restoration protocol includes the insertion of threaded titanium bars into pre-drilled holes in the body of the structural members. The adhesion between marble and bars is achieved by an initially liquid cementitious material. There are two main aspects on which this project focuses. Firstly, the weak link of the marble-cementtitanium complex, the marble-cement interface, is inaccessible for traditional sensing techniques. In this context, the Acoustic Emission technique is employed in order to detect failure and damages at the interior of the complex. Secondly, the specimens and the experimental procedure should simulate the same stress conditions in which all three phases within the marble-cement-titanium complex are under, while the structural member is in service. For this purpose, two modified tests are proposed aiming at efficiently simulating the bars' behavior as they are sliding through a marble epistyle. These tests provide promising results with regards to keeping the examined surface between bars and marble free from parasitic stresses caused by the experimental set-up.
\end{abstract}

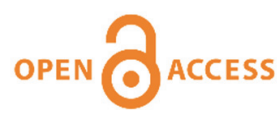

Citation: Dakanali, I., Marinelli, A., An experimental study on restoration technique for the Acropolis of Athens: developing the experimental set-up for pull-out testing of titanium bars embedded in marble blocks, Frattura ed Integrità Strutturale, 50 (2019) 370-382.

Received: 18.02 .2019

Accepted: 28.05.2019

Published: 01.10.2019

Copyright: (C) 2019 This is an open access article under the terms of the CC-BY 4.0, which permits unrestricted use, distribution, and reproduction in any medium, provided the original author and source are credited.

KEYWORDS. Marble; Stone monuments; Pull-out; Restoration; Contact interfaces; Acoustic Emission.

\section{INTRODUCTION}

$\mathrm{T}$ he main restorative intervention on the Athenian Acropolis monuments was directed by N. Balanos in 1885. The interventions were on a grand scale and their purpose was to restore the monuments to the form that they had in classical times. The basic principle was all the destroyed ancient fragments spread around the monument to be re- 
positioned on the monument and the addition of new elements to be limited as much as possible. From that moment, the term "anastylosis" was established as a reconstruction technique whereby a ruined monument is restored using the original architectural elements to the greatest degree possible. From an archeological point of view, the intervention by Balanos was successful as the monument was preserved without largely altering its condition as a ruin (due to the less additions of new marble). Unfortunately, from a structural and chemical point of view, using contemporary - for the time - material without verifying compatibility with the ancient material or chemical resistance against the extensive air pollution, while using scattered ancient fragments as ordinary building material, caused significant damage to the monument [1].

The establishment of the Committee for the Conservation of the Acropolis Monuments (ESMA) in 1975 inaugurated a new approach to the restoration of the monuments of the Acropolis of Athens and tried to heal the injured monument [1]. The committee developed a pioneering technique for the restoration of the monuments' structural integrity according to which the fractured marble elements are connected by inserting titanium bars into pre-drilled holes. The adhesion between marble and bars is achieved by a suitable white cement paste [2]. The aforementioned technique is based on some basic principles: i) Reversibility (if needed, the monument could be brought to its state prior to the intervention), ii) Minimization of the interventions to the extent that guarantees protection of the authentic material from further damage (for this reason the number of titanium bars required by the connection's design, should be the smallest possible) and finally iii) Compatibility between the materials used for the restoration and the authentic ones [3].

After the structural elements are repositioned to their original place, the loads that have to be sustained are their selfweight, the weight of overlying architectural members and the potential impending dynamic loads (using an increasing safety factor). The main goal is to assure that none of the assembled materials gets to be fractured. The only potential failure can be the bars' sliding relatively to the marble. This phenomenon is the so-called 'pull-out'.

The pull-out phenomenon is a matter of concern for the engineers in many structural problems. It has been examined thoroughly in concrete structures. The testing specimens for extracting steel bars or reinforcing fibers from a concrete block have been improved lately in the direction of isolating the tested area from the influence of the experimental set-up. The main drawback of the typical concrete pull-out tests (Fig.1a) is that the stress field of the tested area is not the same with the respective one of the reinforced concrete member. In most concrete structures, the bar and the surrounding concrete are under tension (bending beam) while in pull-out tests, the bar is in tension and the concrete around it is in compression [4]. For this reason, several alternative test designs were proposed (Fig.1(b, c)).

(a)

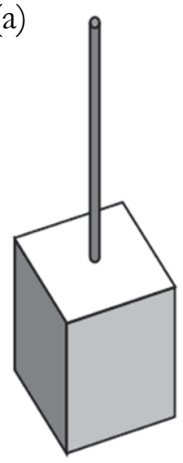

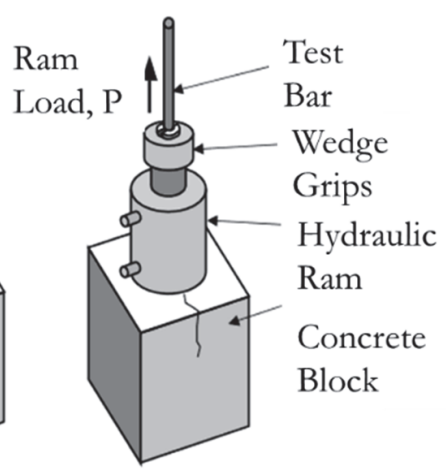

(b)

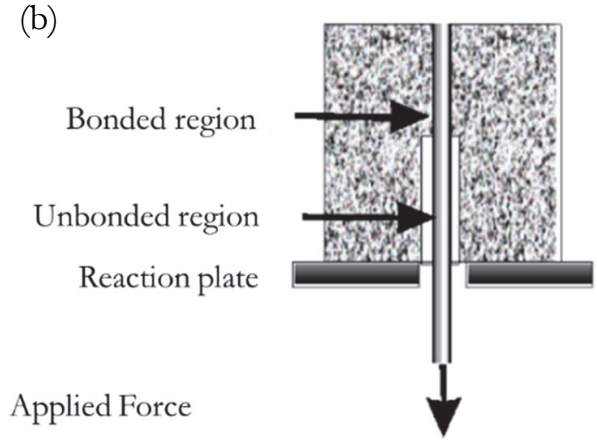

RILEM / CEB/ FIP (1978)

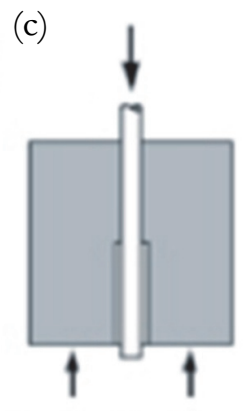

Push in

Figure 1: (a) Typical pull-out specimen [5]; (b) Alternative pull-out test [6]; (c) Push-in concrete specimen [7].

The present work is part of a wider research project for investigating the parameters influencing the pull-out phenomenon in restored marble structural elements [8-10]. As there is no standard protocol for testing the connection used for the restoration of fragmented marble epistyles, the typical pull-out tests of concrete structures have been adopted.

The main goal is not only to simulate experimentally the actual stress field of the assembled parts but also to isolate the intermediate surface between the bars and the marble from the experimental set-up. The concern in monuments after restoration work takes place, is how through thorough monitoring of the structure and 'on time' intervention, there will be no stress field excess.

In most cases in structures, before the final failure, there are microfractures to their interior which are invisible. When collapse is about to occur, there is no time for intervention. Consequently, the continuous monitoring of the structural condition of monuments, considering their sensitivity and cultural importance, is of paramount importance. In this direction, the Acoustic Emission (AE) technique is proposed as a combined innovative non-destructive health monitoring method. 


\section{THE EXPERIMENTAL TECHNIQUE AND ITS APPLICATION}

\section{Acoustic Emission}

$\mathrm{W}$

hen a structure is under stress and the stress field exceeds the materials' capacity, an abrupt release of energy occurs producing transient elastic waves that propagate through the material in the form of concentric circles. The elastic waves propagate inside the material and they can be detected by AE sensors attached on the structure's surface [11]. The acoustic emission technique has three significant advantages. It can locate the damage, provide information for the nature of the acoustic sources and quantify the damage. The accurate source location is an extremely complicated task. The main reasons are that the signal travels in different modes and has a range of velocities. There are also materials that have different velocities in different directions within their volume. Moreover, there are many defects in a material and in most cases the waves are reflected, which causes superpositions or attenuations. Consequently, when the signal is recorded by a sensor it has already been subjected to many alterations [12].

Based on the AE characteristics, useful information can be extracted for the source's nature. Continuous emissions produced by friction [13] have completely different recordable characteristics compared with the burst signals produced by the initiation and propagation of cracks [11]. The basic advantages of the AE method are its high sensitivity, the early detection of defects and cracks and the real-time monitoring at a relatively low cost [14].

Nowadays, an alternative approach is widely used for the classification of the acoustic emissions' source based on the relation between the signals' average frequency with the RA (Rise Time/Amplitude) parameter. The Rise Time RT, is the time interval between the first threshold crossing and the signal peak (Fig.2b). The Rise Time is used for the qualification of signals and as a criterion for noise filter. The amplitude is the greatest measured voltage in a waveform and it is measured in decibels $(\mathrm{dB})$. This is an important parameter in acoustic emission inspection because it determines the detectability of the signal.

The signals with high average frequency and low RA parameter are attributed to tensile cracks. Otherwise, signals with low frequency and higher RA are caused by shear or mixed mode cracks. So far, the results of the qualification of the damage by AE parameters have been proved very encouraging [15-17].

(a)

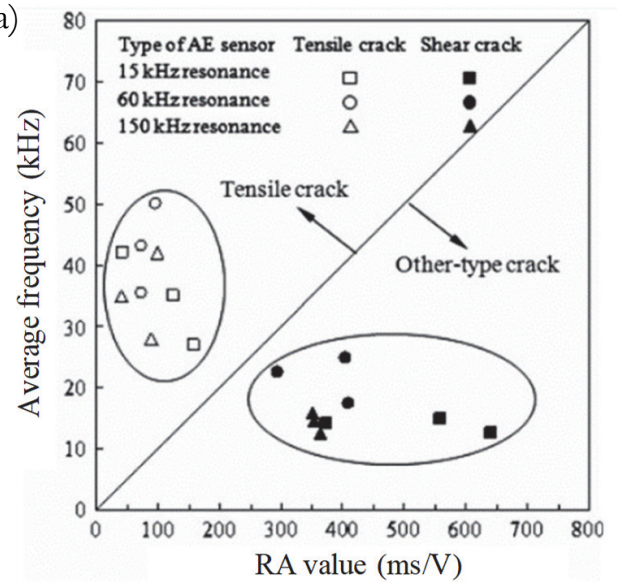

(b)

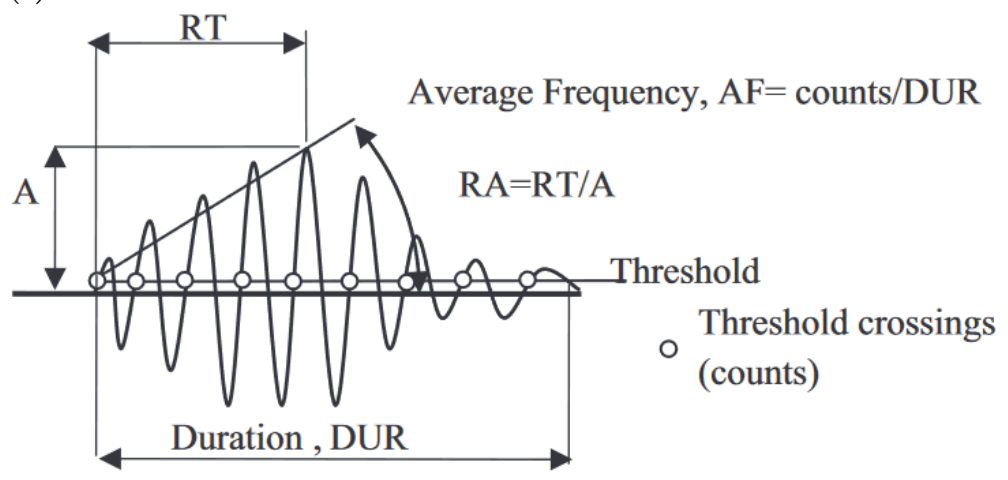

Figure 2: (a) Qualification of the damage by AE parameters [15]; (b) Parameters of an acoustic signal [16].

\section{Experimental procedure}

Three types ('classes') of specimens were prepared. The basic specimens' configuration consists of a marble block $(10 \mathrm{~cm} \mathrm{x}$ $10 \mathrm{~cm} \times 15 \mathrm{~cm}$ ) with a central through hole (diameter:14mm) (Fig.3a). Half of the predrilled hole is filled with a liquid cement paste (binder and water without any aggregate) (Fig.3b). The other half of the hole remains empty. To ensure that, a soft cloth is inserted into the hole preventing the liquid from escaping till its final curing. Meanwhile, a threaded titanium bar is driven into the paste for an appropriate length of the hole (Fig.4c). After removing the cloth, the bar-paste subassemblage is visible into the hole, either at the bottom end of the specimen or half way through its height depending on the specimen's class, providing an entry point for monitoring the bar's pull-out.

More specifically, the first class of experiments includes the classical pull-out tests ( $1^{\text {st }}$ class) [18] with the anchoring length being limited to the upper half of the marble's hole (Fig.4c). The marble block is restrained by a rigid metallic plate with a hole (diameter $=5 \mathrm{~cm}$ ) to its center, where the titanium bar is inserted and gripped by the frame's upper jaw (Fig. 4a, b). The plate is supported by 4 stiff threaded steel bars (Fig.4a). 

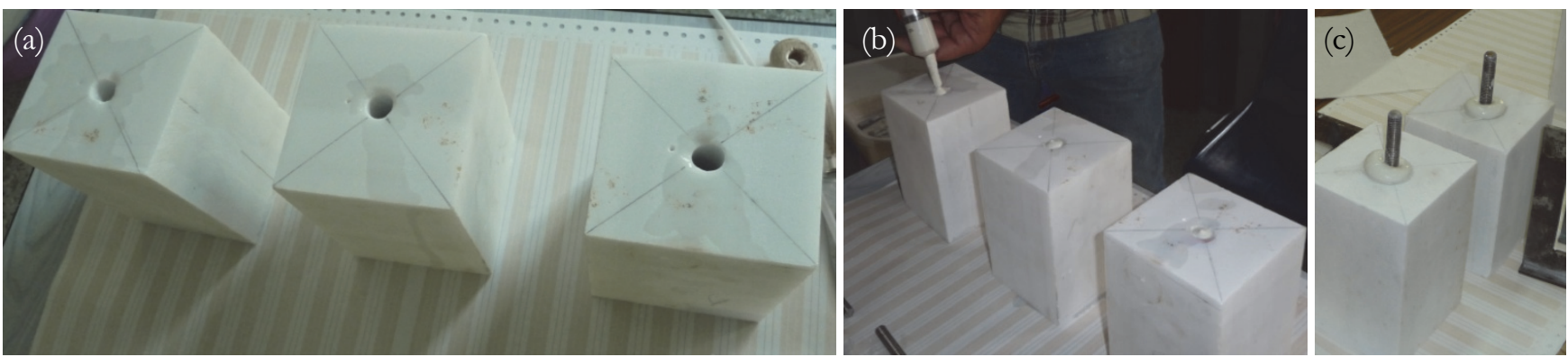

Figure 3: (a) Marble blocks; (b) Filling the hole with cement paste; (c) The titanium bar is driven into the hole.

The second experimental test class ( $2^{\text {nd }}$ class) follows the same main idea. Now the lower half of the hole is filled with an appropriate amount of paste for achieving the same area of connection. The bar is inserted in the full height of the hole, although anchored only for its lower half, with the bar and paste being visible and easily accessible from the bottom end of the specimen (Figs.4c, 5e).

The last class of tests $\left(3^{\text {rd }}\right.$ class) retains the $1^{\text {st }}$ class specimens' configuration but the frame in this case does not pull out the bar but presses it into the marble block. The marble, in this test, is supported on the rigid plate (Fig.4c). The main goal of the alternative specimens and test procedure $\left(2^{\text {nd }}\right.$ and $3^{\text {rd }}$ class) is to isolate the tested area from the supporting system's influence.

All tests were quasi-static, implemented under displacement-control conditions at a rate equal to $0.2 \mathrm{~mm} / \mathrm{min}$. The load was applied monotonically up to the removal of the bar. For the measurement of the axial strain along the bar an InstronDynamic Extensometer of gauge length equal to $12.5 \mathrm{~mm}$ was used. The bar's movement was measured by a calibrated LVDT (Linear Variable Differential Transformer) in contact with the bar's lowest end (Fig.4c). While for the 2nd class of specimens the bar was directly accessible at their bottom surface (Fig.5e), for the $1^{\text {st }}$ and $3^{\text {rd }}$ classes, the LVDT was inserted into the empty part of the through hole, from the bottom surface of the specimen until it reached the bar's lowest end.

For the acoustic emission recordings, 8 acoustic sensors are mounted around the marble near the surface between the paste and the marble, where the potential signals are expected (Fig.5). For comparison purposes, the anchoring length of the bar is $7.5 \mathrm{~cm}$ for all the specimens. The experiments were performed after a 28-days curing period for the paste.
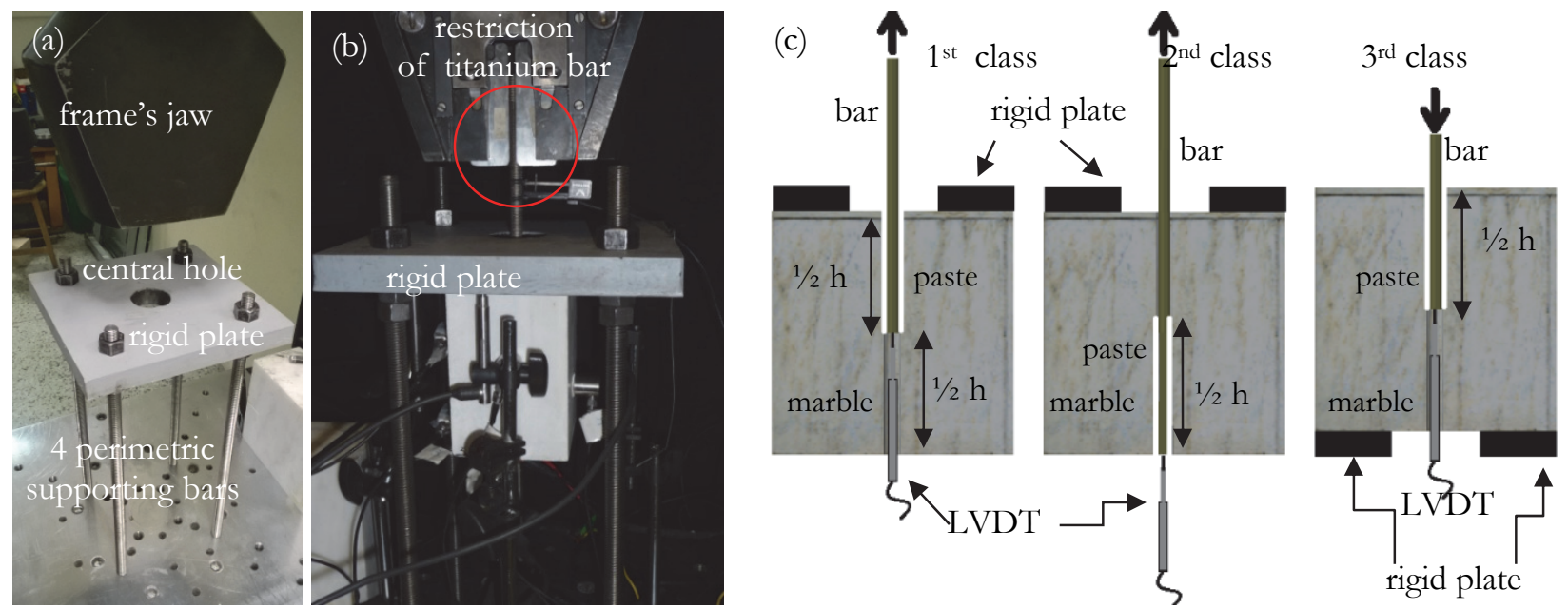

Figure 4: Testing configuration: (a) Rigid plate, supporting bars; (b) marble and titanium bar's restriction; (c) the 3 different experimental approaches.

\section{EXPERIMENTAL RESULTS}

\section{Standard data}

he diagrams in Fig.6 show the time variation of the load for all 3 test classes. The curves of the $1^{\text {st }}$ class tests (Fig. 6a) include a first linear segment. Before reaching the maximum load, the curve changes inclination. It is noticeable that when the connection loses its integrity (maximum load) there is a characteristic load drop. The respective 

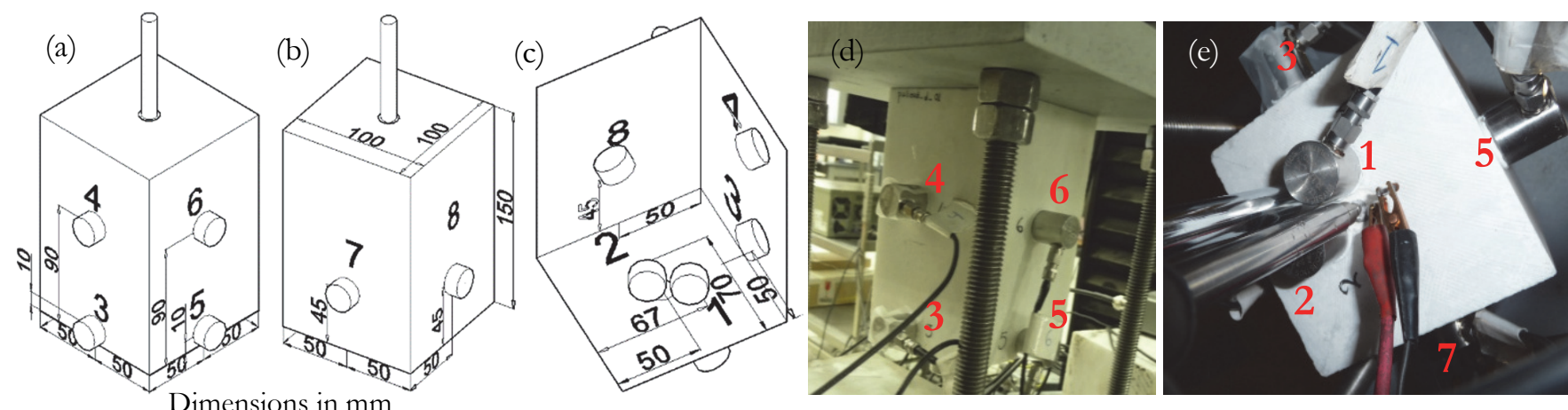

Figure 5: Acoustic sensors around the specimen (2 ${ }^{\text {nd }}$ class).

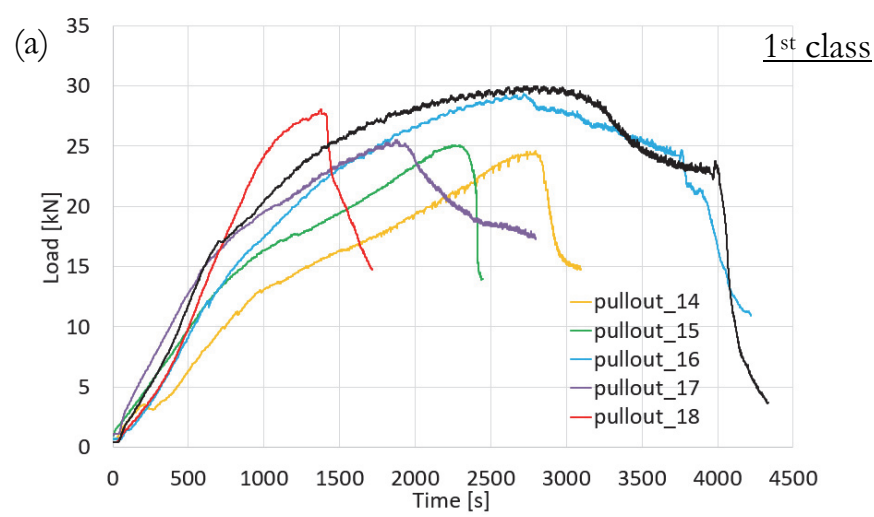

(b)

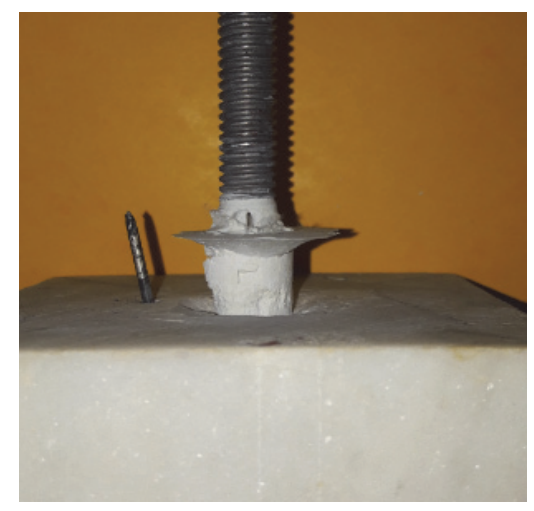

$\underline{2^{\text {nd }} \text { class }}$

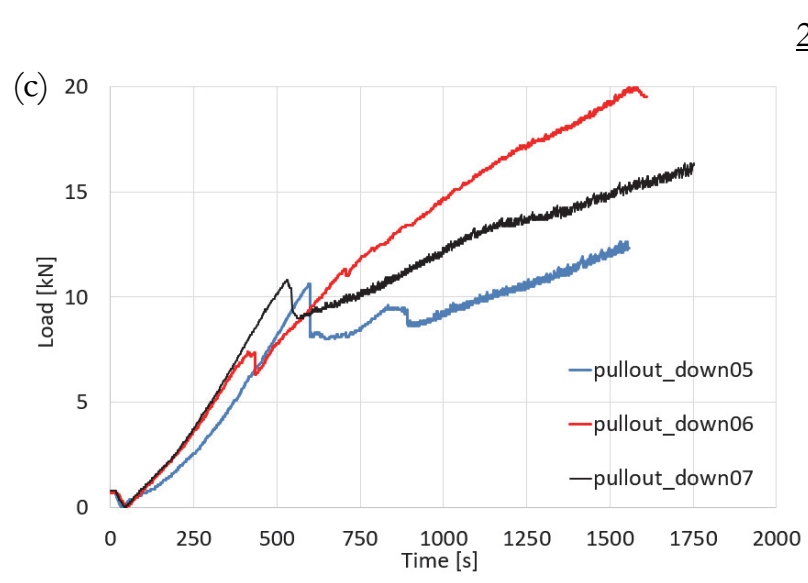

(c) 20

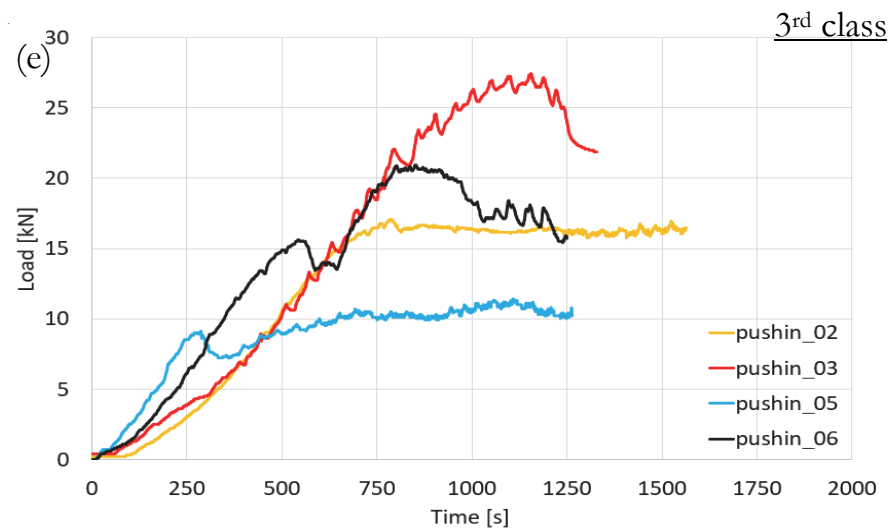

(d)

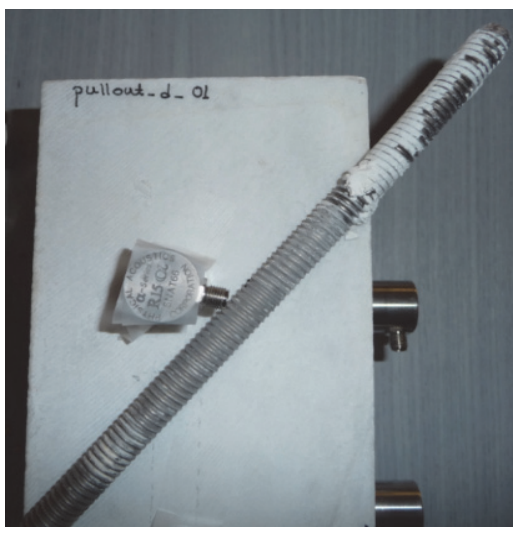

Figure 6: (a); (c); (e) The time variation of the load for the 3 different classes of tests; (b); (d); (g) the specimens after the test; (f) the specimen before the test ( $3^{\text {rd }}$ class). 


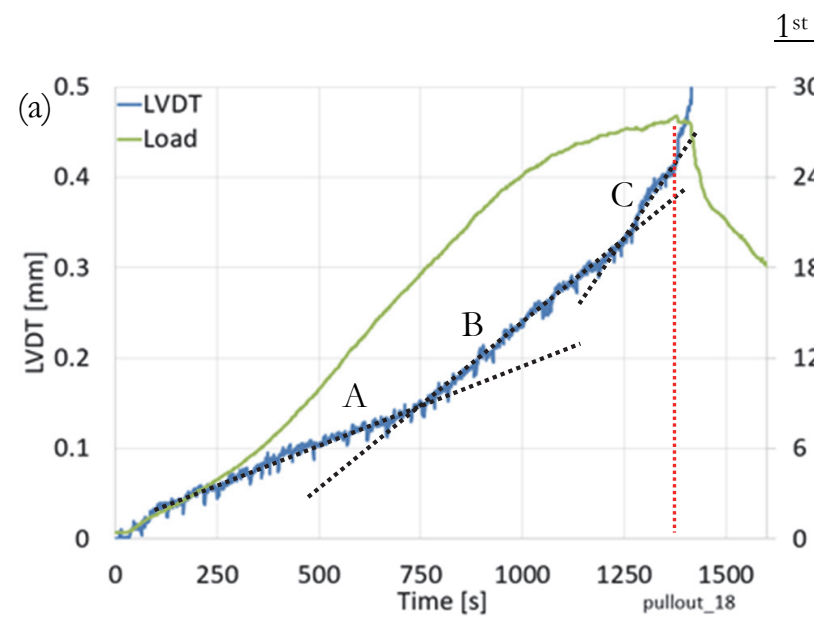

$\underline{1 \text { st } \text { class }}$

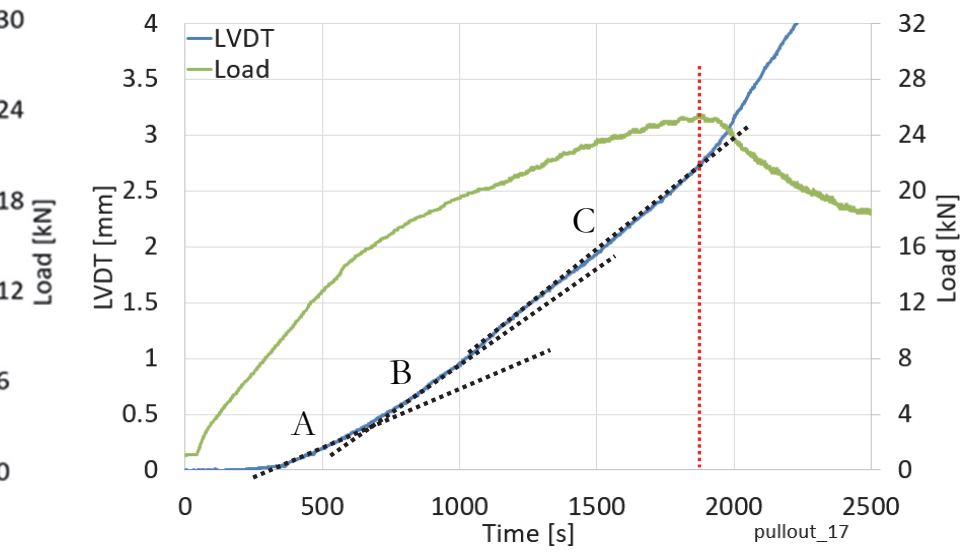

$\underline{2^{\text {nd }} \text { class }}$
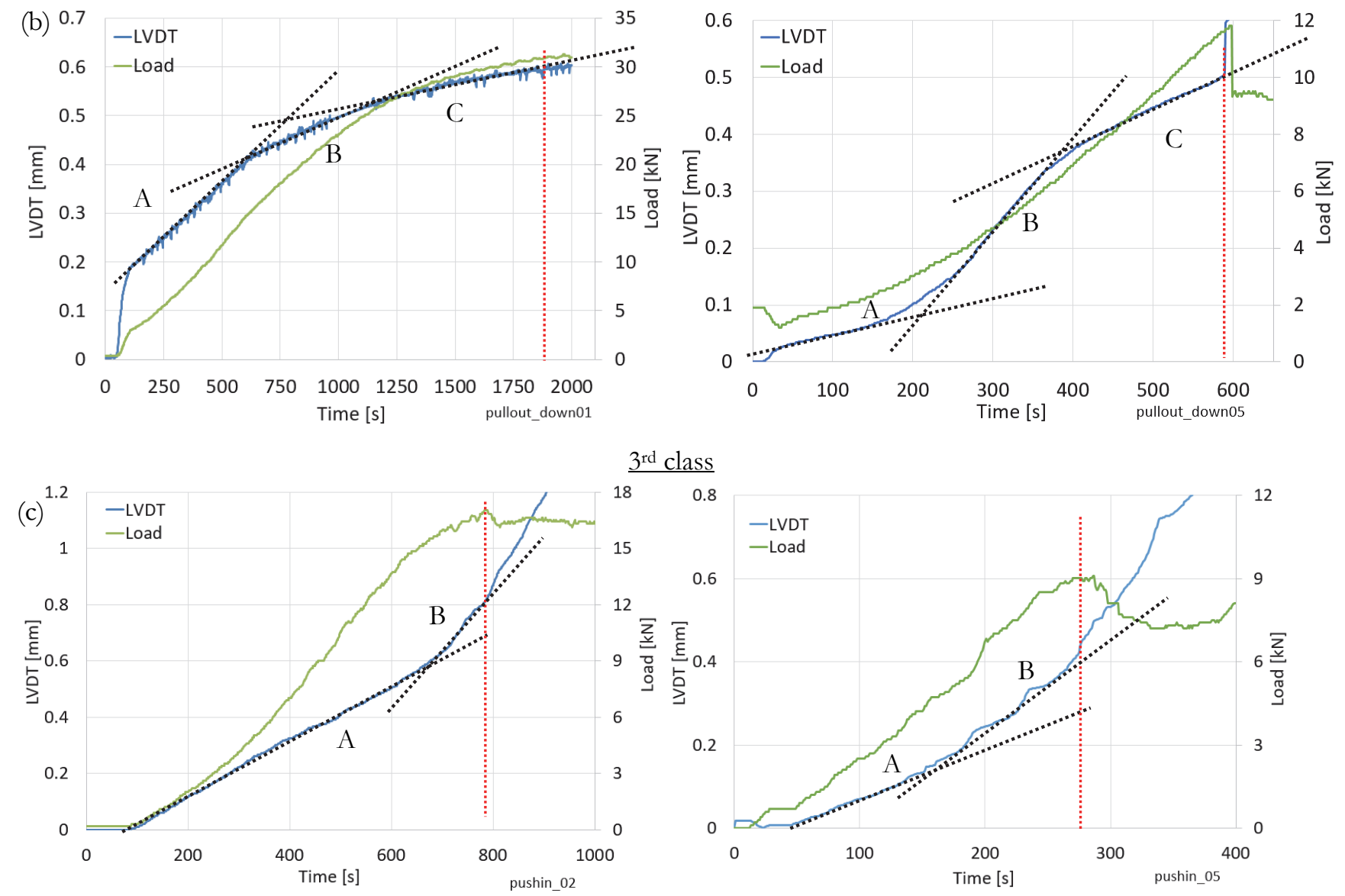

$\underline{3^{\text {rd }} \text { class }}$

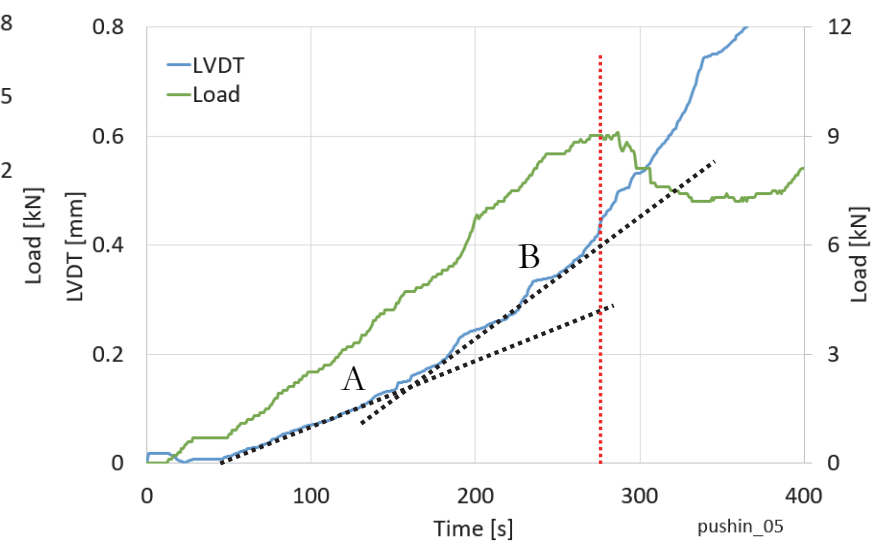

Figure 7: The time variation of the load and the LVDT indications for two characteristic tests of each experimental class.

curves for $2^{\text {nd }}$ class tests are presented in Fig.6c. The curve has a linear segment till the maximum load when a slight load drop occurs. After that, the load continues to increase. This is due to the friction as the bar is sliding through the remaining hole's length. It is important that after the bar's extraction from the marble block, the bars from all $2^{\text {nd }}$ class tests present a characteristic cone of marble around them (Fig.6b), which does not appear after the tests of $2^{\text {nd }}$ class (Fig.6d).

The load from $3^{\text {rd }}$ class tests increases linearly till the maximum load, after that a slight drop appears and the load remains relatively constant. The connection does not lose totally its integrity due to the friction between the paste-bar complex and the marble, as the frame is pushing inwards the bar. 
Differences observed between the curves of all experiments within each class could be well attributed to uncontrollable parameters related to the inhomogeneity and anisotropy of Dionysos marble [19-23], the size effect characterizing its properties $[24,25]$ as well as to the different properties of the paste.

The LVDT indications (blue line) for the bar's movement during the experiments for two characteristic tests of each class are shown in Fig.7. The $1^{\text {st }}$ and $2^{\text {nd }}$ class LVDT curves present three distinct inclination changes before the maximum load. The first two changes are due to the elastic response of the whole experimental system and only the third change is due to the bar's sliding. It is observed that there is a correlation between the load curve and the respective LVDT's curve. It also seems that the load vs. time curve could be sensitive to the experimental configuration's boundary conditions and specific specimen details, which leads to variations on the behavior recorded (Fig.7). For example, for $1^{\text {st }}$ and $2^{\text {nd }}$ class, three changes of inclination are generally observed but for the second test in $2^{\text {nd }}$ Class (Fig.7b) there is an initial concave upward LVDT curve followed by a concave downward part. The respective curves of two characteristic push-in tests (Fig.7c) show that the LVDT indications' rate changes slightly before the maximum load.

\section{Data from innovative sensing techniques}

A thorough analysis of the data gathered from the AE sensors reveals that the acoustic emission recordings are in temporal correlation with the changes of the bar's displacement and the load. This correlation can be seen in Fig.8, in which the time variation of the duration and energy of the AEs are plotted versus time, in conjunction with the respective time variation of the data gathered from the LVDT in contact with the bar's lowest end, for a $1^{\text {st }}$ class typical test. In the same figure, the variation of the load versus time is plotted (green line). It is evident that the AEs before the bar's sliding are fewer and of lower energy and duration compared to the respective AEs of the 3rd part, after the sliding starts.

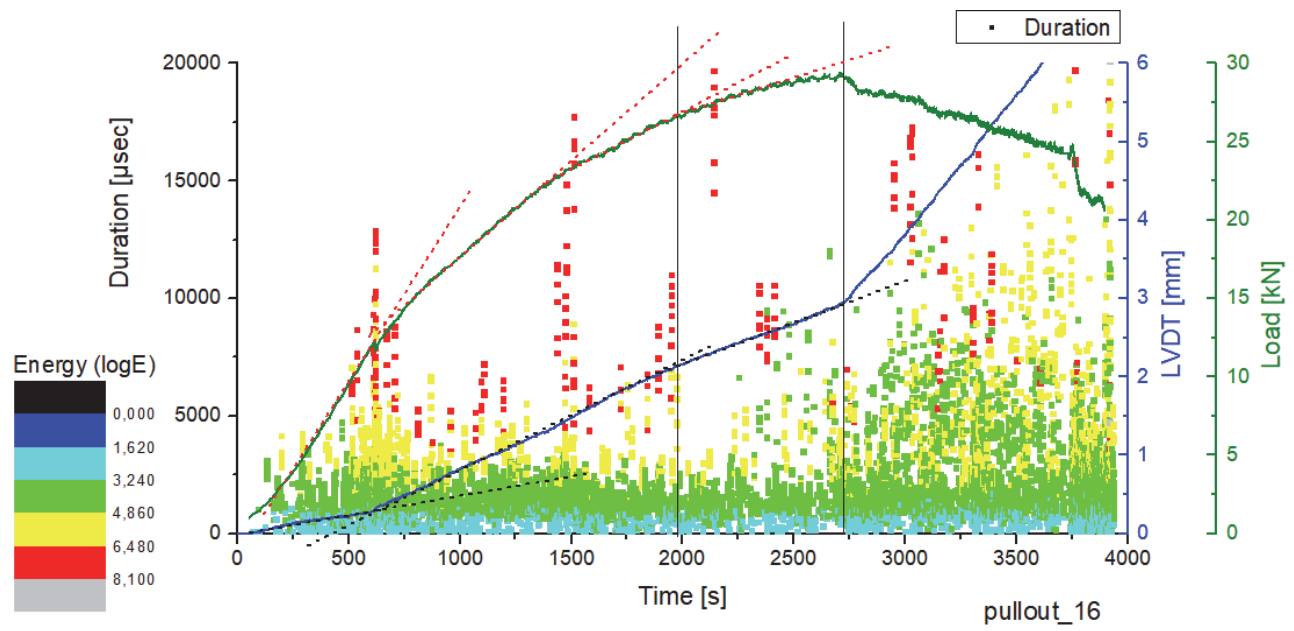

Figure 8: ' 1 st Class' test: time variation of the load and the LVDT indications with the duration and energy (contour plot) of the AEs.

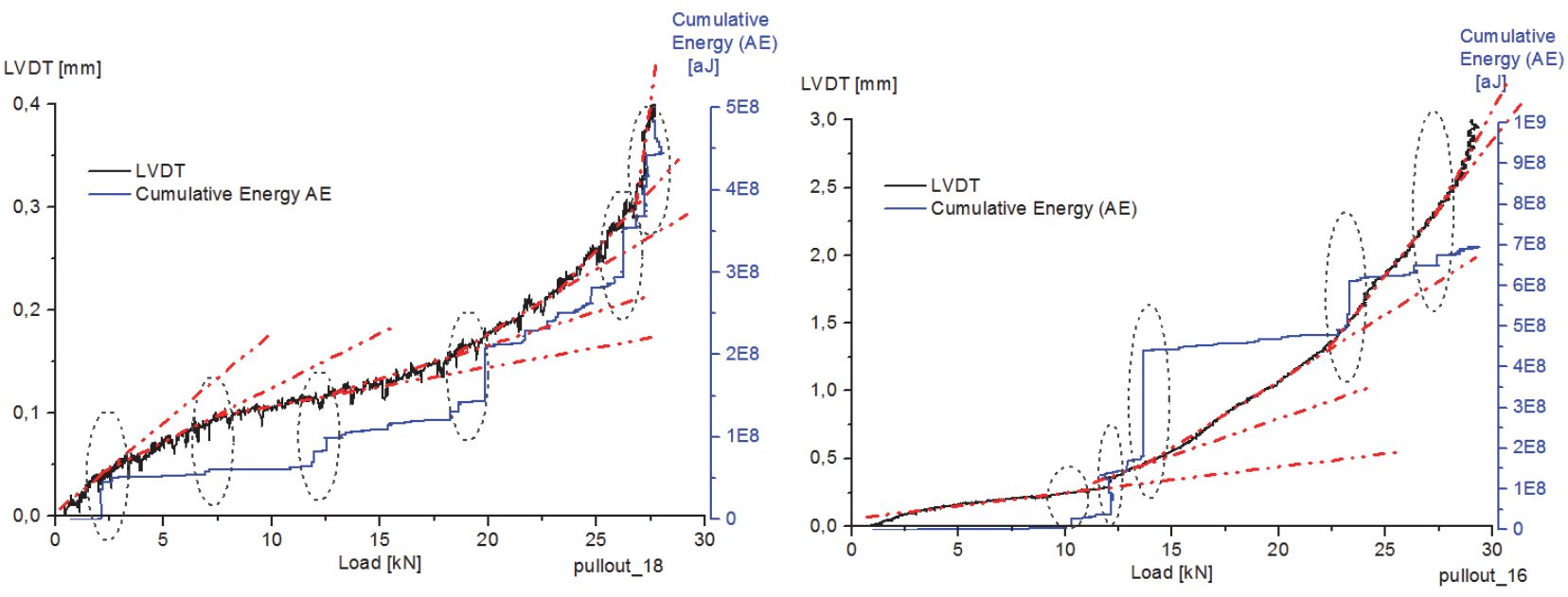

Figure 9: '1 1 st Class' tests: variation of the bar's displacement and cumulative energy of the AE versus load. 
Moreover, the characteristic slope changes of the load-time curve are accompanied by increased AEs duration. After the maximum load, when the connection has lost its integrity, there are many acoustic signals of high duration that are attributed to the prevailing co-existing friction as the bar is sliding through the hole.

The variation of the bar's displacement and that of the cumulative energy of the AE versus the load induced are plotted in Fig.9 for two typical experiments ( $1^{\text {st }}$ class). It is very interesting to observe that almost simultaneously with every slope change of the bar's displacement curve, a significant increase of the energy of the AEs is recorded. Despite the fact that the $1^{\text {st }}$ class pull-out tests give very encouraging AE results, it is obvious that the acoustic signals produced by the experimental set-up dominate the final results and the pure pull-out events are 'covered' by those parasitic events. Considering that the alternative design of the pull-out tests ( $2^{\text {nd }}$ class) focuses on the tested area, the results are expected to be clearer.

The time variation of the duration and energy of the AEs, the load and the data gathered from the LVDT in contact with the bar's lowest end for a typical $2^{\text {nd }}$ class test are plotted in Fig.10a. It is obvious that during the first two periods regarding the changes of inclination for the LVDT curve, there is not a significant number of acoustic signals. The accumulation of the AEs started slightly before the third slope change of the LVDT curve. The location of the Acoustic Events and specifically their " $y$ " position along the bar-paste area versus time is plotted in Fig.10b. The system started to record AE along the anchoring length when the LVDT's indications curve changes inclination for the third time before the maximum load.

Considering that the above results are clearer and most of the parasitic signals have been removed, it could be useful to categorize the recorded signals produced due to the pull-out phenomenon. Based on the average frequency and RA parameter analysis $[16,17]$ the respective diagrams for two characteristic $2^{\text {nd }}$ class tests are drawn (Fig. 11). There are four time ranges, three according to the LVDT curve inclination's change and one after the maximum load. The first and second area's signals (A\&B) presented a significant decrease of the RA parameter. The third area $(C)$ presents a worth mentioning

(a)

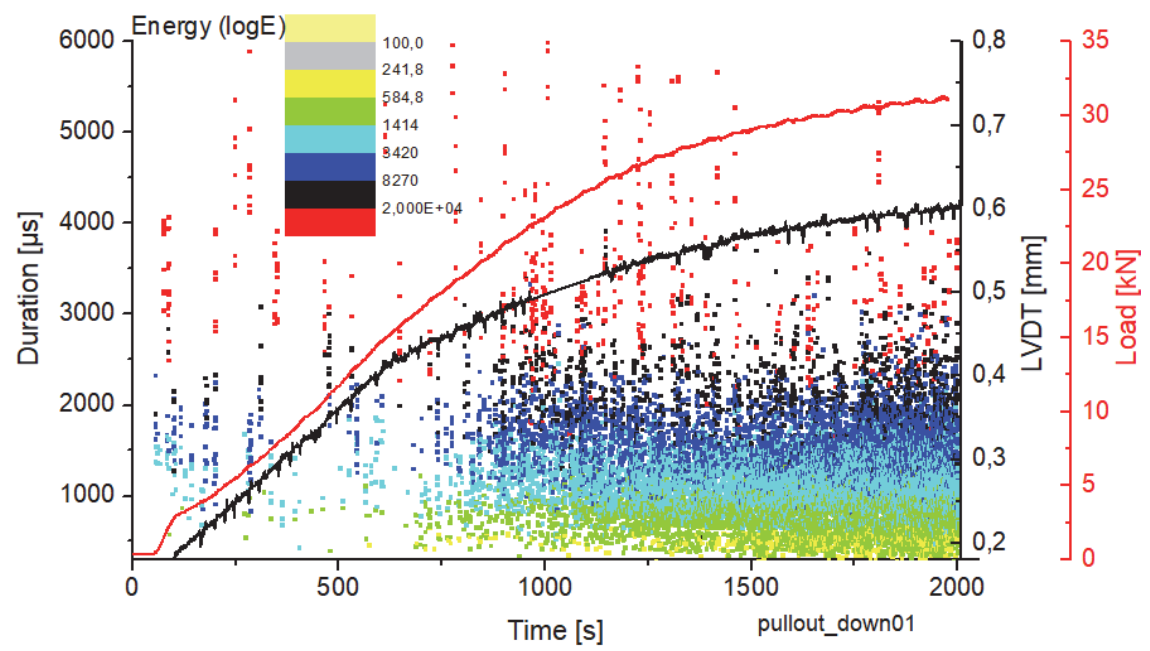

(b)

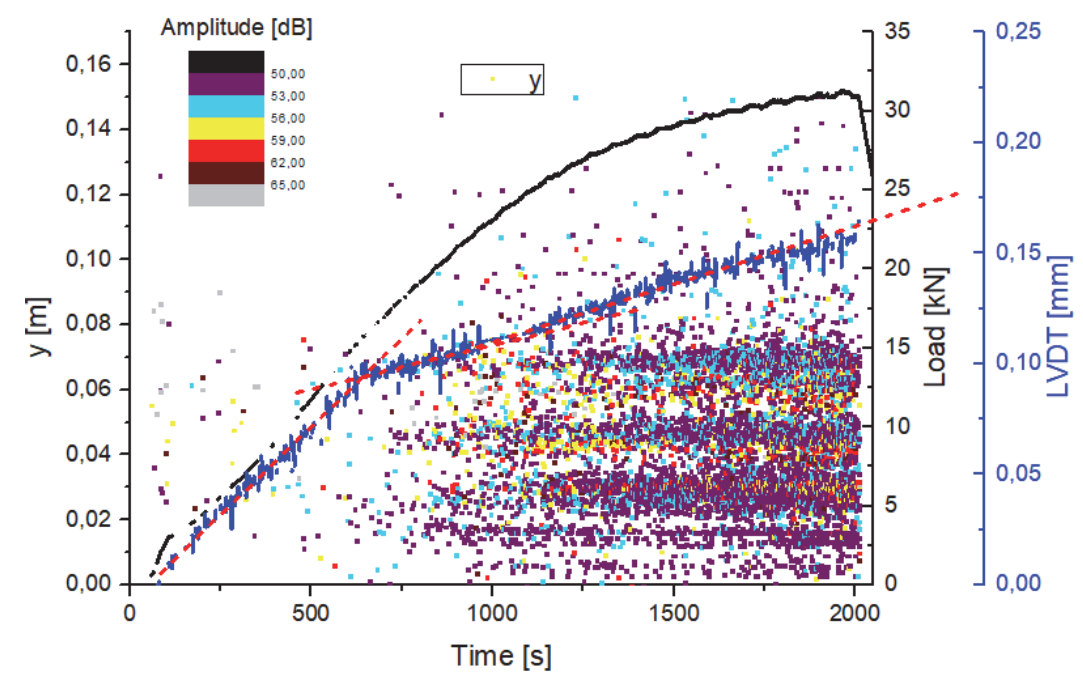

Figure 10: '2 $2^{\text {nd }}$ Class' test: (a) Time variation of the load and the LVDT indications with the duration and energy (contour plot) of the AEs; (b) Time variation of the LVDT indications, the load and the y position of the AEs (along the anchoring length). 

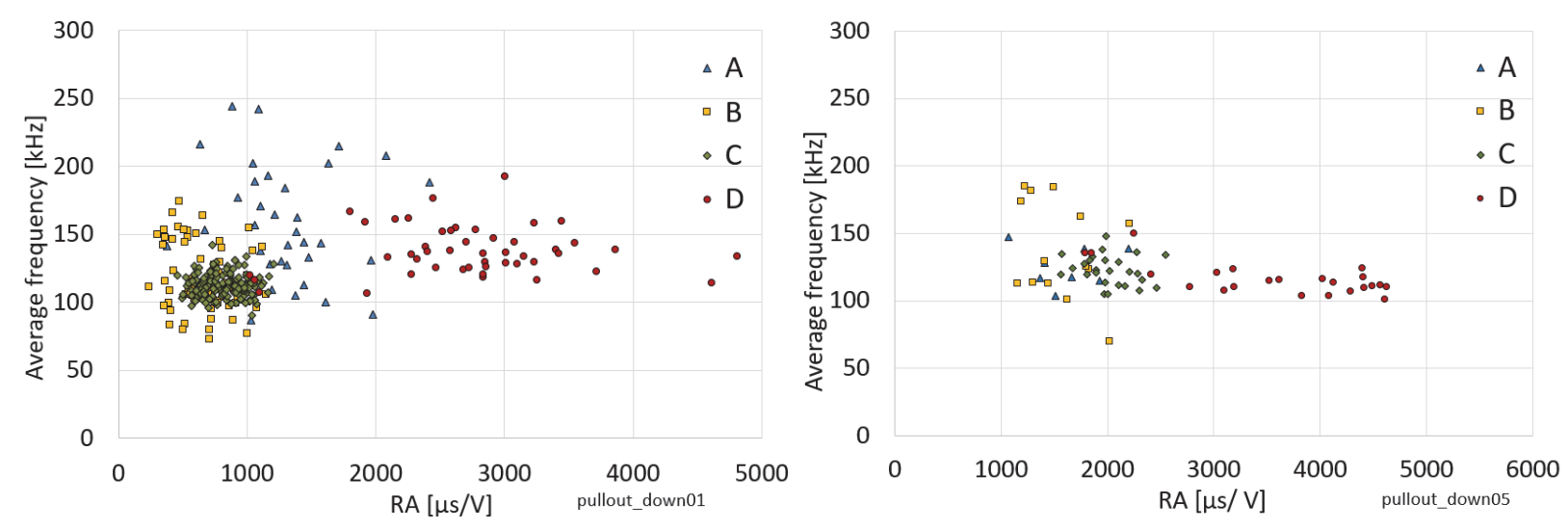

Figure 11: Average frequency versus RA parameter for two typical '2 2 nd $C l a s s$ ' tests.

accumulation. The signals with low RA parameter are attributed to tensile cracks and this is because of the paste's cracks and the connection's failure. After the maximum load, the signals present an increased RA parameter. It is evident that in this time most of the signals are due to shear or mix mode fracture (the prevailing bar's sliding).

In Fig.12, the time variation of the duration and energy of the AEs, the load and the data gathered from the LVDT of a characteristic $3^{\text {rd }}$ class test are plotted. Significant number of AEs is recorded from the very beginning. This is also clear from the AE location through the anchoring length in Fig.12b. When the bar is under compression, the paste around experiences more cracks due to bar's expansion.

From the diagram of the average frequency versus the RA parameter for the two characteristic time periods before the maximum load of two push-in experiments (Fig.13), it is evident that the cracks increase as the parameter RA decreases from $\mathrm{A}$ to $\mathrm{B}$ time range.
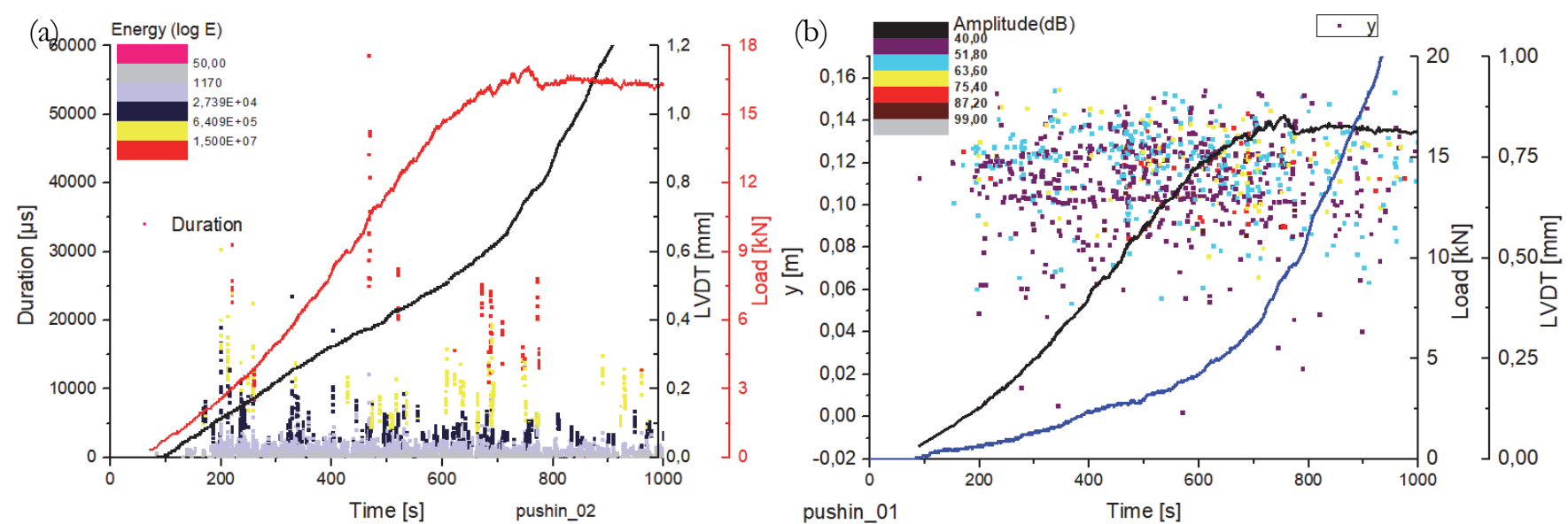

Figure 12: ' $3^{\text {rd }}$ Class' test: (a) Time variation of the load and the LVDT indications with the duration and energy (contour plot) of the AEs; (b) Time variation of the LVDT indications, the load and the y position of the AEs (along the anchoring length).
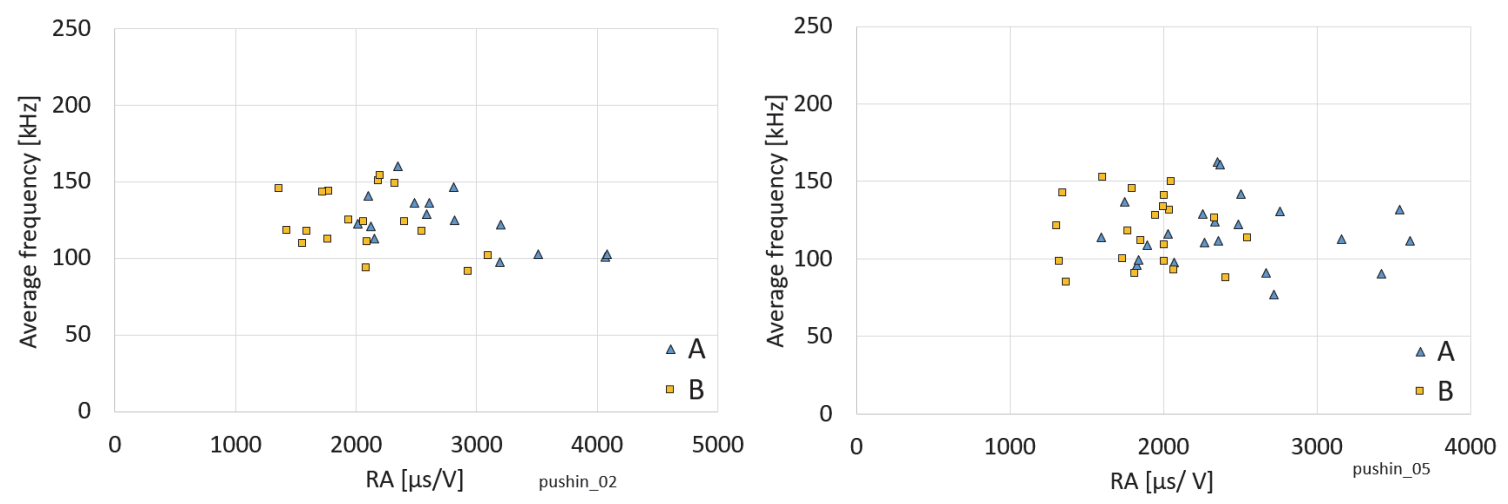

Figure 13: Average frequency versus RA parameter for two typical ‘ 3 rd Class' tests. 


\section{ONGOING WORK - INNOVATIVE EXPERIMENTAL CONFIGURATIONS}

$\Lambda$ lthough the last examined configurations provided encouraging results and it seems that the AE technique managed to detect the onset of the bar's sliding, the parasitic signals were not minimized to the least. For this reason, the specimens are modified again in order to find the proper configuration that focuses perfectly on the pull-out phenomenon, not only in this case (marble-paste-bar) but in any bar extraction (concrete - reinforcement bar).

The first new alternative approach includes two pieces of marble. A hole of the same depth and diameter is precisely drilled in both marble blocks. The two pieces are connected with a titanium bar and cement paste, following exactly the same procedure for the restoration of fragmented marble blocks as in the Acropolis' monuments (Figs.14(a,b)). During the experimental procedure, the lower marble piece is restricted with the rigid metallic plate and the upper piece is forced to move upwards so that the bar slides through the hole (Fig.14c).

The second alternative test configuration includes a piece of marble with two holes in the upper and lower edge of the specimen. A bar is inserted to each end (Figs.15(a,b)). The bottom bar is restricted by the lower jaw of the testing frame while the upper one holds the top bar. The upper jaw moves upwards forcing the bars to be pulled-out while the lower jaw remains still (Fig.15). The anchoring lengths at both ends are identical.

The bar that was pulled-out was the lower one, while the upper one remained intact in position (Fig.15d). Given the early stages of this investigation, it is to be further studied which of the two bars would be pulled-out at a number of repetitions of the test and under which circumstances.

(a)

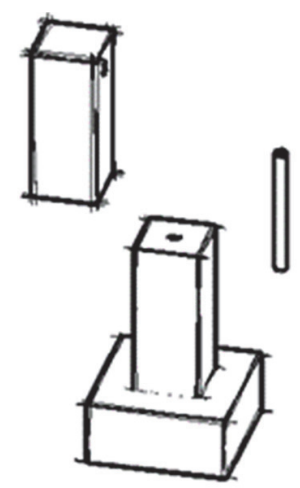

(b)

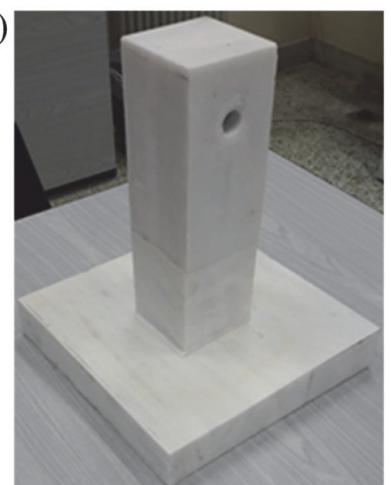

(c)

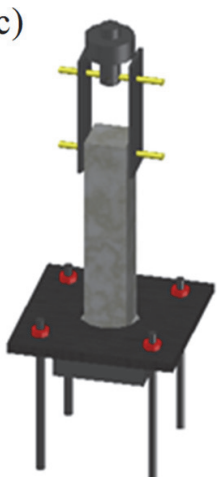

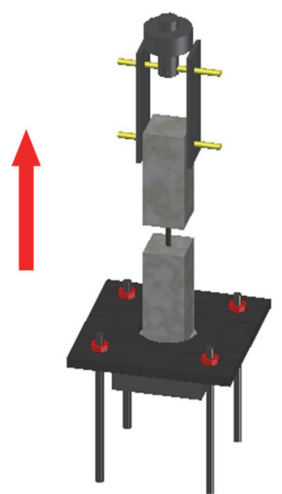

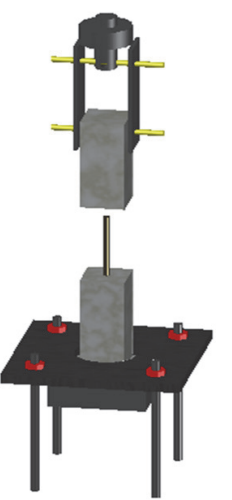

Figure 14: (a) $1^{\text {st }}$ suggested alternative configuration: the pieces of the specimen, marble blocks and titanium bar (schematically); (b) marble assembly; (c) test configuration (positioning and test procedure).
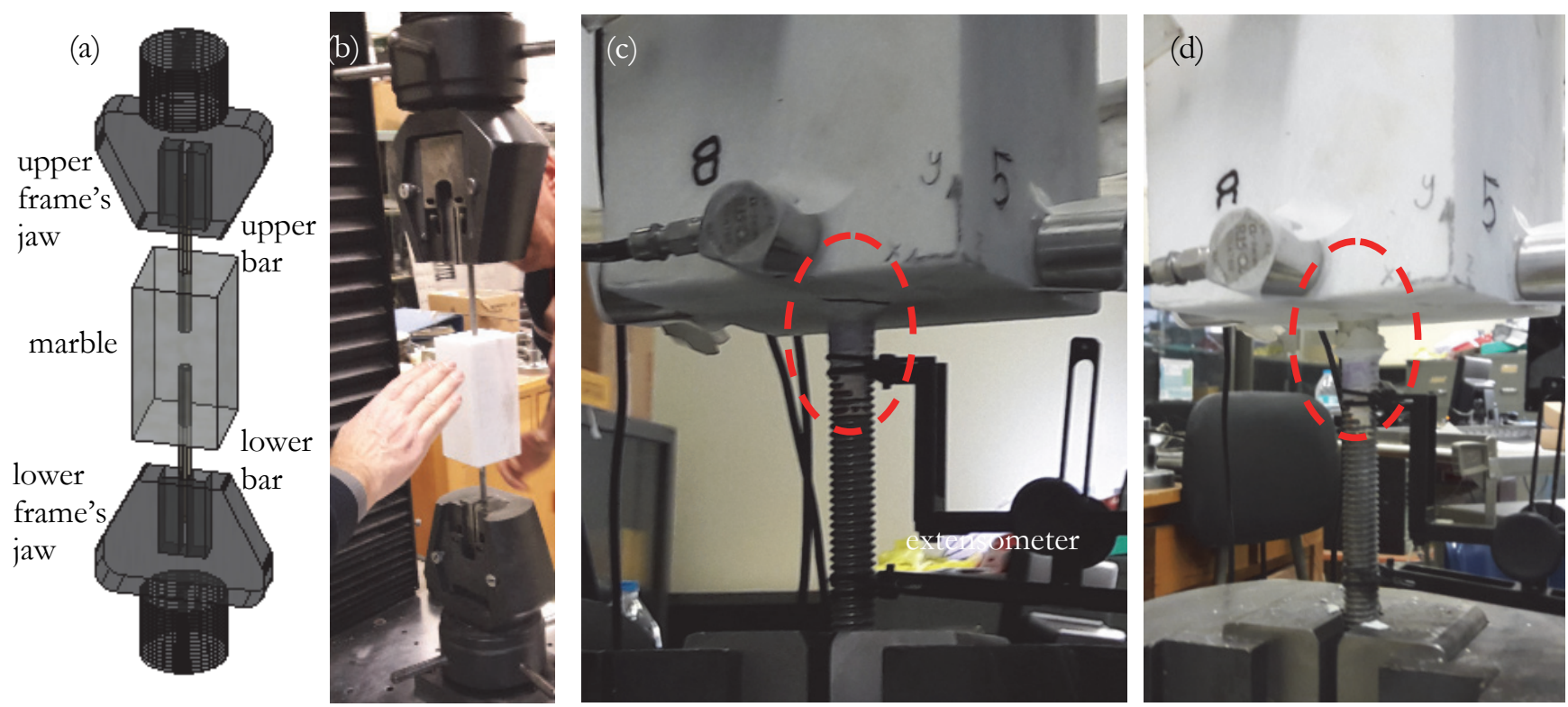

Figure 15: (a) $2^{\text {nd }}$ suggested alternative test configuration (schematically); (b) test procedure; (c) the onset of the lower bar's extraction; (d) detail of the failure mode after testing. 
According to the raw data from the first attempt of the double pull-out configuration (Fig.16a), it seems that the ultimate pull-out load is lower than that in other pull-out tests (Fig.16b). A possible explanation could be that the marble does not interfere with the pull-out of the bar and the frame's load is opposed only by the strength and cohesion of the marblepaste interface. The bar's deformation assumes also lower values compared to the other two occasions (pull-out $1^{\text {st }}$ class, pull-out $2^{\text {nd }}$ class) and this could prove that the assembly parts (marble, bar) are not under stress, with the only active part being the interface between marble and cement paste (Fig.16b). From a different perspective (Fig.16c), in an effort to "neglect" the maximum load's influence and focus on the load changes for the three different tests, the ratio Load/ maxLoad is provided versus the bar's strain. The curve's inclination and the bar's strain for the double pull-out are noticeably larger than those of the other two (1 $1^{\text {st }}$ and $2^{\text {nd }}$ class).
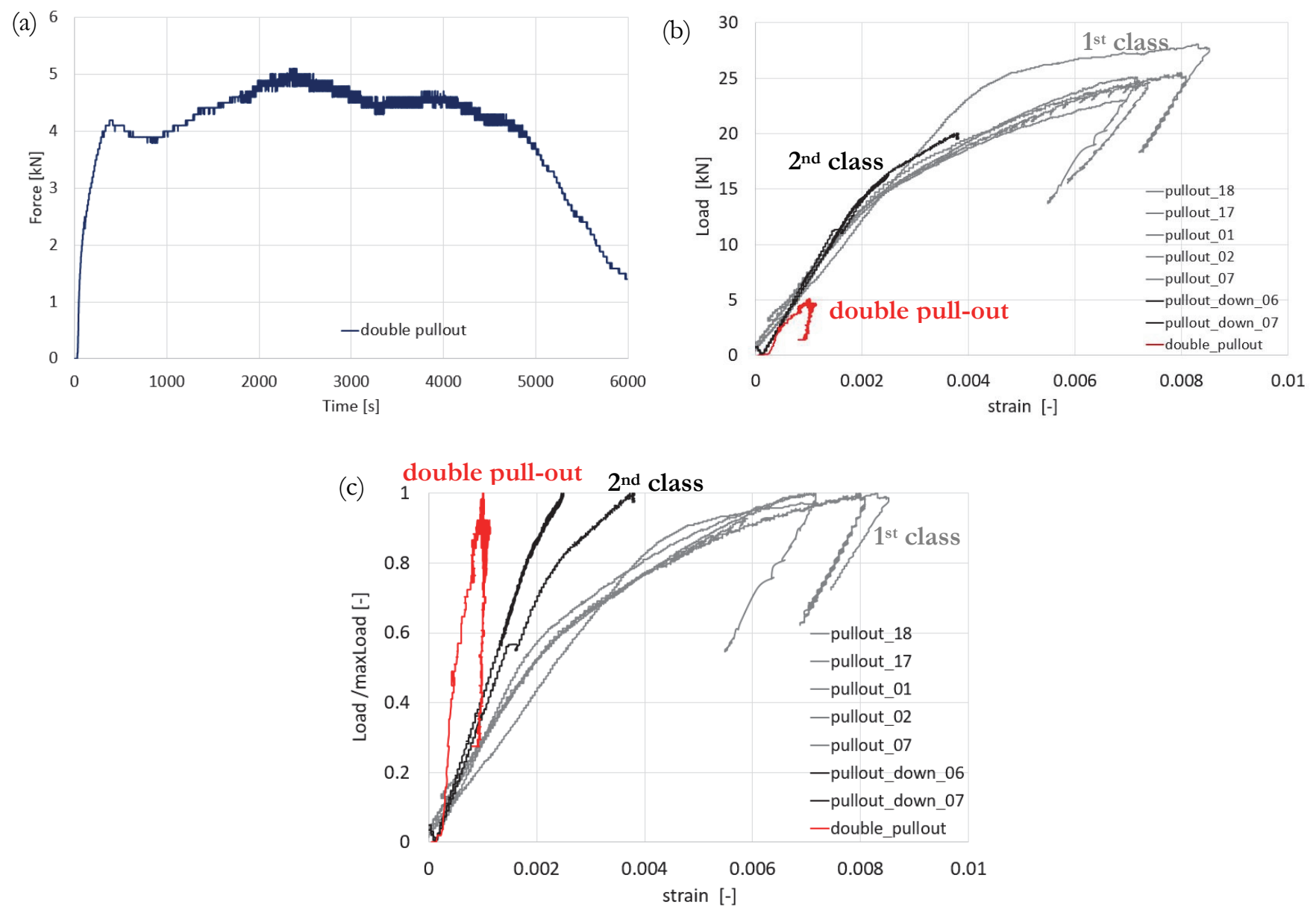

Figure 16: (a) Time variation of load (double pull-out test); (b) Load vs bar's strain for pull-out test (1 ${ }^{\text {st }}$ class), pull-out down (2 ${ }^{\text {nd }}$ class) and the double pull-out test; (c) Load/maxLoad vs bar's strain for pull-out test (1 ${ }^{\text {st }}$ class), pull-out down (2 ${ }^{\text {nd }}$ class) and the double pull-out test.

\section{CONCLUSIONS}

C

urrent research in the field of restoration of the Acropolis monuments addresses the quality of adhesion between marble and reinforcing bars used to rejoin fragmented elements. According to the restoration perception, it is of paramount importance that the ancient marble pieces are protected and not further fractured because of an insufficient connection. Experience from restoration projects when the restored structural member is subjected to tension and bending, stresses the importance of investigating the failure mechanism leading to the debonding and finally to the catastrophic pull-out of the reinforcing bar from the body of the marble volume. Under certain loading conditions, the pull-out phenomenon dominates the failure mode, for which further study is required even though there is some standardization of the experimental procedure. 
This study focuses on the quantification of the role of parasitic stresses, developed due to the technique used to restrict and load the specimens, having as a case study the 'marble-cement paste-titanium bar' complex appearing during the restoration of marble epistyles of the Parthenon temple.

For this purpose, Dionysos marble prisms are tested in a variety of configurations ( 3 different classes), having a threaded titanium bar centrally placed and connected by means of suitable cement paste. Pulling-out and pushing-in of the bar are considered in juxtaposition. Innovative sensing techniques (AE) permitting detection of failure and damages at the interior of the complex are used, together with standard measuring and recording equipment. The time variation of the load for the 3 classes of tests, together with the corresponding LVDT measurements, constitute the basic dataset of the study, providing an insight to the evolution of failure modes through changes of curves' inclination before the maximum load. The AE sensors' measurements versus time indicate when sliding starts and reveal characteristics of the failure modes, via observed changes in the number, energy and duration of recordings.

Experimental results seem to be strongly influenced by the load application procedure as well as by the support conditions, indicating the need to reconsider standard testing configurations. Modifications to the initially adopted ones are suggested aiming at finding an experimental set-up that focuses perfectly on the pull-out phenomenon, not only in this case (marblepaste-bar) but in any bar extraction (concrete-reinforcement bar). Preliminary results from two alternative test configurations and procedures are promising since they are compatible with the failure mode being dependent only from the strength and cohesion of the interface between the marble and the cement paste.

\section{REFERENCES}

[1] Lebidaki, E., (2011). The restoration of the monuments of the Athenian Acropolis, $2^{\text {nd }}$ ed., Ministry of culture and tourism-acropolis restoration service, Athens.

[2] Korres, M., Toganides, N., Zambas, C. and Skoulikidis, Th. (1989). Study for the restoration of the Parthenon, Ministry of Culture, Committee for the Preservation of the Acropolis Monuments (in Greek), Vol.2a, Athens.

[3] Acropolis Restoration Service. (2011). Principles and Methods. Available at: http://www.ysma.gr/en/restorationprinciples-and-methods, (accessed 10/01/2017).

[4] Looney, T.J., Arezoumandi, M., Volz, J.S. and Myers, J.J. (2012). An experimental study on bond strength of reinforcing steel in self consolidating concrete, International Journal of Concrete structures and Materials. 6 (3), pp 187-197. DOI: $10.1007 /$ s40069-012-0017-9

[5] Thompson, M.K., Jirsa, J.O., Breen, J.E. and Klingner, R.E. (2002). Bond and development length of deformed bars. Austin: Texas, Department of Transportation.

[6] Achillides, Z. and Pilakoutas, K. (2004). Bond behavior of fiber reinforced polymer bars under direct pullout conditions, Journal of Composites for Construction, 8(2), pp. 173-181. DOI: 10.1061/(asce)1090-0268(2004)8:2(173)

[7] Alvarez, M. (1998). Einfluss des Verbundverhaltens auf das Verformungsvermögen von Stahlbeton. ETH Zürich, Diss., Basel: Birkhäuser (IBK Bericht 236). DOI: 10.3929/ethz-a-002000033

[8] Kourkoulis, S.K., Papanicolopulos, S.-A., Marinelli, A. and Vayas, I., (2008). Restoration of antique temples: Experimental investigations on the pull-out behaviour of anchors in marble, Bautechnik, 85(2), pp 109-119.

DOI: 10.1002 / bate.200810010

[9] Dakanali, I., Stavrakas, I., Triantis, D. and Kourkoulis, S.K. (2016). Pull-out of threaded reinforcing bars from marble blocks. Procedia Structural Integrity, 2, pp 2865-2872. DOI: 10.1016/j.prostr.2016.06.358

[10] Kourkoulis, S.K., Marinelli, A. and Dakanali, I. (2016). A combined experimental and numerical study of the pull-out mechanism of threaded titanium bars embedded in marble blocks, ECCOMAS Congress 2016 - VII European Congress on Computational Methods in Applied Sciences and Engineering, M. Papadrakakis, V. Papadopoulos, G. Stefanou, V. Plevris (eds.), Crete Island, Greece. DOI: 10.7712/100016.2174.8066

[11] Grosse, C.U. and Ohtsu, M., (2008). Acoustic Emission testing, Basics for research - Applications in Civil Engineering, Springer-Verlag Berlin Heidelberg. DOI: 10.1007/978-3-540-69972-9

[12] Kaphle, M. (2012). Analysis of Acoustic Emission data for accurate damage assessment for structural health monitoring applications. (Doctoral dissertation), Queensland University of Technology.

[13] Miller, RK., McIntyre, P. (1987). NDT handbook-acoustic emission testing, Columbus, OH: American Society for Nondestructive Testing.

[14] Mistras group Hellas ABEE, http://mistrasgroup.gr/acoustic_emission_theory_eng.htm (accessed 10/01/2017).

[15] Ohtsu, M. (2010). Recommendation of RILEM TC 212-ACD: acoustic emission and related NDE techniques for crack detection and damage ecaluation in concrete. Materials and Structures, 43, pp 1187-1189.

DOI: $10.1617 / \mathrm{s} 11527-010-9639-z$ 
[16] Aggelis, D.G. (2011). Classification of cracking mode in concrete by acoustic emission parameters. Mechanics Research Communications, 38, pp 153-157. DOI: 10.1016/j.mechrescom.2011.03.007

[17] Ohno, K., Ohtsu, M. (2010). Crack classification in concrete based on acoustic emission, Construction and Building Materials, 24, pp 2339-2346. DOI: 10.1016/j.conbuildmat.2010.05.004

[18] Marinelli, A., Papanicolopulos, S.-A., Kourkoulis, S.K., Vayas, I. (2009). The pull-out problem in restoring marble fragments: a design criterion based on experimental results. Strain, 45, pp 433-444.

DOI: 10.1111/j.1475-1305. 2008.00519.x

[19] Kourkoulis, S.K., Exadaktylos, G. and Vardoulakis, I. (1999). U-notched Dionysos Pentelicon marble in three point bending: The effect of non-linearity, anisotropy and microstructure, International Journal of Fracture, 98, pp. 369-392. DOI: $10.1023 / \mathrm{a}: 1018614023542$

[20] Exadaktylos, G., Vardoulakis, I. and Kourkoulis, S.K. (2001). Influence of nonlinearity and double elasticity on flexure of rock beams - II. Characterization of Dionysos marble, International Journal of Solids and Structures, 38 (22-23), pp. 4119-4145. DOI: 10.1016/S0020-7683(00)00252-3

[21] Vardoulakis, I., Kourkoulis, S.K., Exadaktylos, G. and Rosakis, A. (2002). Mechanical properties and compatibility of building stones of ancient monuments: Dionysos marble. Proc. of the Interdisciplinary Workshop "The building stone in monuments", Athens, IGME Publishing, Eds. M. Varti- Mataranga and Y. Katsikis, pp 187-210.

[22] Kourkoulis, S.K., Ganniari-Papageorgiou, E. and Mentzini, M. (2010). Dionysos marble beams under bending: A contribution towards understanding the fracture of the Parthenon architraves, Engineering Geology, 115(3-4), pp. 246-256. DOI: 10.1016/j.enggeo.2009.06.012.

[23] Kourkoulis, S.K., Prassianakis, I., Agioutantis, Z. and Exadaktylos, G.E. (2006). Reliability assessment of the NDT results for the internal damage of marble specimens, International Journal of Material and Product Technology, 26(1/2), pp. 35-56.

[24] Kourkoulis, S.K. and Ganniari-Papageorgiou, E. (2010). Experimental study of the size- and shape-effects of natural building stones, Construction and Building Materials, 24(5), pp. 803-810. DOI: 10.1016/j.conbuildmat.2009.10.027.

[25] Vardoulakis, I., Exadaktylos, G.E. and Kourkoulis, S. K. (1998). Bending of marble with intrinsic length scales: A gradient theory with surface energy and size effects, Journal de Physique IV, 8, pp. 399-406. DOI: $10.1051 /$ jp4: 1998849. 